\title{
The Magnetic Fields in the Ophiuchus and Taurus Molecular Clouds
}

\section{Citation}

Troland, T. H., R. M. Crutcher, A. A. Goodman, C. Heiles, I. Kazes, and P. C. Myers. 1996. “The Magnetic Fields in the Ophiuchus and Taurus Molecular Clouds." The Astrophysical Journal 471 (1): 302-7. https://doi.org/10.1086/177970.

\section{Permanent link}

http://nrs.harvard.edu/urn-3:HUL.InstRepos:41397392

\section{Terms of Use}

This article was downloaded from Harvard University's DASH repository, and is made available under the terms and conditions applicable to Other Posted Material, as set forth at http:// nrs.harvard.edu/urn-3:HUL.InstRepos:dash.current.terms-of-use\#LAA

\section{Share Your Story}

The Harvard community has made this article openly available.

Please share how this access benefits you. Submit a story.

Accessibility 


\title{
THE MAGNETIC FIELDS IN THE OPHIUCHUS AND TAURUS MOLECULAR CLOUDS
}

\author{
T. H. Troland, ${ }^{1}$ R. M. Crutcher, ${ }^{2}$ A. A. Goodman, ${ }^{3}$ C. Heiles, ${ }^{4}$ I. Kazès, ${ }^{5}$ AND P. C. Myers ${ }^{6}$ \\ Received 1995 July 20; accepted 1996 May 14
}

\begin{abstract}
We discuss the significance of previously published $\mathrm{OH}$ Zeeman effect measurements of the magnetic field in the $\rho$ Oph cloud (L1688) and also a new OH Zeeman effect measurement for the dark cloud core TMC-1C, the latter obtained with the Arecibo telescope. Results for both of these clouds, like previously published $\mathrm{OH}$ Zeeman results for other dark clouds, show that the line-of-sight field strength $\left|B_{\|}\right| \leq 10$ $\mu \mathrm{G}$. The $\rho$ Oph cloud is significantly higher in column density than other clouds studied, and recent $\mathrm{H}_{\mathrm{I}}$ Zeeman effect observations have revealed $B_{\|} \approx+10 \mu \mathrm{G}$ in the $\mathrm{H}$ I self-absorption region in front of the cloud. The low values of $B_{\|}$derived from the $\mathrm{OH}$ Zeeman effect may indicate that $\mathrm{OH}$ poorly samples the dense gas in the $\rho \mathrm{Oph}$ cloud, or else that the cloud is in a state of supercritical collapse. The TMC-1C cloud core may have formed via ambipolar diffusion in a manner similar to recent modeling of dark cloud Barnard 1.
\end{abstract}

Subject headings: ISM: clouds — ISM: individual (rho Ophiuchi, Taurus) - ISM: magnetic fields ISM: molecules - polarization - stars: formation

\section{INTRODUCTION}

It has long been realized that many aspects of selfgravitating molecular clouds are difficult to understand in the absence of magnetic fields. For example, it is not clear how these clouds can be supported against gravity in the absence of magnetic fields nor how they can rid themselves of angular momentum as they form new stars. Some of these issues are outlined in reviews by Mouschovias (1987, 1991a, 1991b), Shu, Adams, \& Lizano (1987), and McKee et al. (1993).

With these considerations in mind, we have carried out an extensive program of Zeeman effect observations in the $18 \mathrm{~cm}$ (1665 and $1667 \mathrm{MHz}) \mathrm{OH}$ emission lines from dark clouds (Crutcher et al. 1993, hereafter Paper I). These observations were obtained with the NRAO $43 \mathrm{~m}$ telescope ${ }^{7}$ having a beam width of $18^{\prime}$, large enough to encompass the cloud cores as well as their envelopes. The Zeeman effect is only sensitive to $\boldsymbol{B}_{\|}$, the component of $\boldsymbol{B}$ parallel to the line of sight. The measured value of $B_{\|}$at any particular position is an average over the telescope beam, so reversals of field direction within the beam can lessen the observed effect. Of 12 dark cloud positions reported upon in Paper I, only one yielded an unambiguous detection. This position was toward the dark cloud Barnard 1, for which we measured $B_{\|}=-19 \pm 4 \mu \mathrm{G}$. (Positive fields are directed away from the observer.) Taken as a whole, the results of Paper I are consistent with an average total field strength of $|\boldsymbol{B}|=16 \mu \mathrm{G}$ in the regions sampled by these observations.

\footnotetext{
${ }^{1}$ Department of Physics and Astronomy, University of Kentucky, Lexington, KY 40506.

${ }^{2}$ Department of Astronomy, University of Illinois, Urbana, IL 61801.

${ }^{3}$ Department of Astronomy, Harvard University, 60 Garden Street, Cambridge, MA 02138.

${ }^{4}$ Department of Astronomy, University of California, Berkeley, CA 94720.

${ }^{5}$ Observatoire de Paris-Section de Meudon, Place Jules Janssen, F-92195 Meudon, France.

${ }^{6}$ Harvard Smithsonian Center for Astrophysics, 60 Garden Street, MS 42, Cambridge, MA 02138.

7 The National Radio Astronomy is operated by Associated Universities, Inc. under cooperative agreement with the National Science Foundation.
}

In this paper, we discuss two of these positions toward the $\rho$ Oph cloud (L1688), and we also present new OH Zeeman effect data obtained with the Arecibo telescope for the dark cloud core TMC-1C. The $\rho$ Oph positions are of special interest for several reasons. For one, the $\rho$ Oph cloud has much higher column and volume densities than the other dark cloud positions reported in Paper I, hence it might be expected to have stronger magnetic fields as well. Also, because of the higher densities involved, observations of the $\rho$ Oph cloud may bear upon the question of $\mathrm{OH}$ abundance at higher densities and the role of $\mathrm{OH}$ Zeeman observations in revealing field strengths in higher density regions. Finally, H I Zeeman effect measurements have recently been reported for the $\rho$ Oph region by Goodman \& Heiles (1994), so a comparison of field strengths sampled by $\mathrm{OH}$ and $\mathrm{H}$ I in the same cloud can now be made. Such comparisons have also been possible for the molecular cloud associated with S106 (Roberts, Crutcher, \& Troland 1995). The TMC-1C result is of interest because it was obtained at much higher spatial resolution $\left(3^{\prime}\right)$. Moreover, preliminary evidence reported by Güsten \& Fiebig (1990) suggested a magnetic field of order $100 \mu \mathrm{G}$ might exist in this core.

\section{OBSERVATIONS AND RESULTS}

Observations of the $18 \mathrm{~cm} \mathrm{OH} \mathrm{Zeeman} \mathrm{effect} \mathrm{toward} \mathrm{the}$ $\rho$ Oph cloud are reported in Paper I as positions $\rho$ Oph Nos. 1 and 2, and the observational techniques with the 43 $\mathrm{m}$ telescope are discussed in that paper along with possible instrumental effects. Likewise, very similar observational techniques employed with the Arecibo telescope are described by Goodman et al. (1989). An essential difference between Zeeman effect observations of the two clouds considered here is telescope beamwidth. Almost certainly, the larger $43 \mathrm{~m}$ beam width $\left(18^{\prime}\right)$ samples primarily the envelope regions of the clouds, while the smaller Arecibo beam width $\left(3^{\prime}\right)$ samples to a greater extent the cloud cores.

Figure 1 shows the Stokes $I$ and $V$ spectra for the $\rho$ Oph cloud position $1\left(\alpha_{1950} 16^{\mathrm{h}} 24^{\mathrm{m}} 16^{\mathrm{s}}, \delta_{1950}-24^{\circ} 27^{\prime} 00^{\prime \prime}\right)$. This position coincides with the densest part of the ridge of $\mathrm{C}^{18} \mathrm{O}$ emission in the map of Wilking \& Lada (1983). From the 


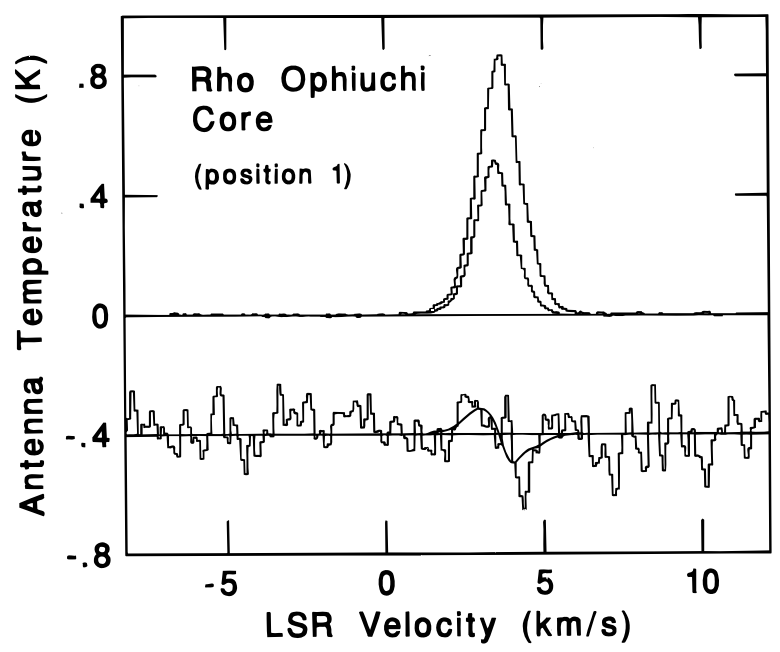

FIG. 1.-Stokes parameter $I / 2=(R+L) / 2$ spectra for the $1667 \mathrm{MHz}$ (stronger line) and $1665 \mathrm{MHz}$ lines of $\mathrm{OH}$ toward $\rho$ Oph core position 1 , shown with baselines at zero, and the average Stokes parameter $V=R-L$ multiplied by 30 and plotted offset from zero, all shown as histograms. The average $V$ spectrum is the average of $V$ spectra for 1665 and $1667 \mathrm{MHz}$ lines. The smooth curves superposed upon the $V$ spectrum is the derivative of the average $I / 2$ spectra, scaled for $B_{\|}=-9.5 \mu \mathrm{G}$, the value determined by a least square fit to the spectra.

data of Figure 1, we derive $B_{\|}=-9.5 \pm 3.0 \mu \mathrm{G}^{8}$ using standard analysis techniques described in Paper I. For $\rho$ Oph position $2\left(\alpha_{1950} 16^{\mathrm{h}} 24^{\mathrm{m}} 30^{\mathrm{s}}, \delta_{1950}-24^{\circ} 10^{\prime} 38^{\prime \prime}\right)$, located about one beamwidth to the northeast of position 1 and off the $\mathrm{C}^{18} \mathrm{O}$ ridge, we find $B_{\|}=+1.3 \pm 4.3 \mu \mathrm{G}$, as reported in Paper I.

In Figure 2 we present Stokes $I$ and $V$ spectra for TMC-1C obtained at Arecibo $\left(\alpha_{1950} 04^{\mathrm{h}} 38^{\mathrm{m}} 31^{\mathrm{s}} .5, \delta_{1950}\right.$ $\left.-25^{\circ} 55^{\prime} 00^{\prime \prime}\right)$. From these data, we derive $B_{\|}=+1.4 \pm 2.4$ $\mu \mathrm{G}$.

\section{THE $\rho$ Oph CLOUD}

\subsection{The Nature of the $\rho$ Oph Cloud}

Most of the dark cloud positions reported in Paper I contain standard cold cloud cores $(T \approx 10 \mathrm{~K})$ that are the centers of low-efficiency low-mass star formation. Averaged over the $18^{\prime}$ beam of the $43 \mathrm{~m}$ telescope, these positions typically have $N_{\mathrm{OH}} \approx 4 \times 10^{14} \mathrm{~cm}^{-2}$ (Paper I, Table 2). Therefore, if $N_{\mathrm{OH}} / A_{V} \approx 8 \times 10^{13} \mathrm{~cm}^{-2} \mathrm{mag}^{-1}$ or $N_{\mathrm{OH}} /$ $N_{p} \approx 4 \times 10^{-8}$ for dark clouds (Crutcher 1979; valid for $0.4<A_{V}<7$ ), then $A_{V} \approx 5 \mathrm{mag}, N_{p} \approx 10^{22} \mathrm{~cm}^{-2}$, and $n_{p} \approx$ few $\times 10^{3} \mathrm{~cm}^{-3}$.

The $\rho$ Oph cloud is significantly different from these more standard dark clouds in a number of ways. At a distance of $125 \pm 25$ pc (de Geus, Zeeuw, \& Lub 1989), it is warmer ( $T$ as high as $30 \mathrm{~K}$; Loren, Sandquist, \& Wootten 1983; Wilking \& Lada 1983; Zeng, Batrla, \& Wilson 1984; de Geus \& Burton 1991), the star formation efficiency is higher, and at least a few B stars are forming along with lower mass stars (Wilking \& Lada 1983; Lada \& Wilking 1984). Gas column densities are also higher. Analysis of $\mathrm{C}^{18} \mathrm{O}$ emission of the $\rho$ Oph cloud with a 1'1 beam led Wilking \& Lada (1983) to conclude that $A_{V}$ reaches $100 \mathrm{mag}$ at the cloud center. In Figure 3 we reproduce their map of $A_{V}$ derived from the $\mathrm{C}^{18} \mathrm{O}$ data assuming $A_{V} / N\left(\mathrm{C}^{18} \mathrm{O}\right)=3.6 \times 10^{-15}$

\footnotetext{
${ }^{8}$ The sign of this field was erroneously reported in Table 2 of Paper I as positive. However, all other magnetic field signs in the table are correct as verified by contemporaneous observations of $\mathrm{OH}$ masers of known circular polarization
}

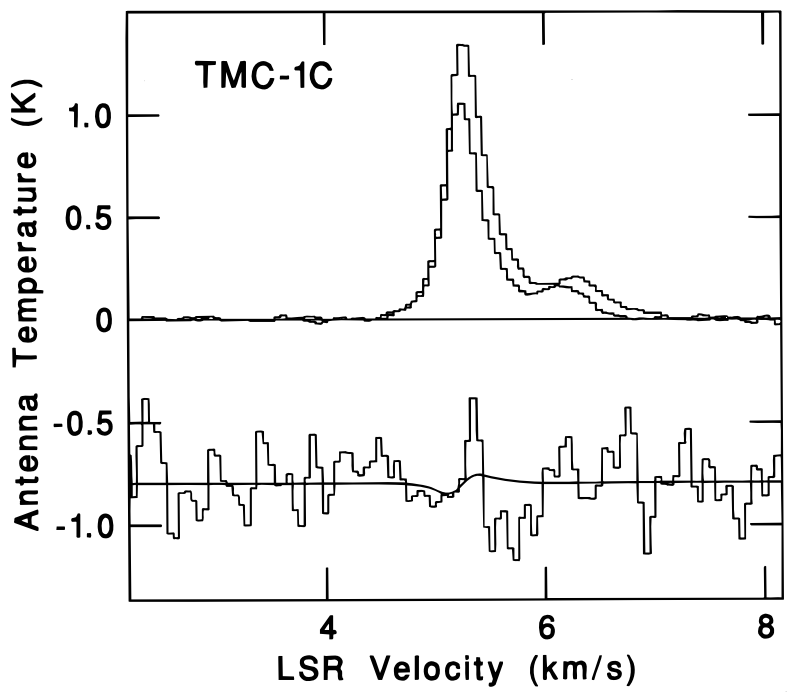

FIG. 2.-A similar display of data as in Fig. 1, except for the dark cloud core TMC-1C. The average of the 1667 and $1665 \mathrm{MHz}$ Stokes $V=R-L$ spectra is multiplied by 20 relative to the Stokes $I / 2$ spectra. The smooth curve superposed upon the $V$ spectrum is the derivative of the average $I / 2$ spectra, scaled for $B_{\|}=+1.4 \mu \mathrm{G}$, the value determined by a least square fit to the spectra.

mag $\mathrm{cm}^{2}$. We have added circles to the $A_{V}$ map indicating the $43 \mathrm{~m}$ telescope beam at $\rho$ Oph positions 1 and 2 .

A visual inspection of Figure 3 indicates $A_{V} \approx 50 \mathrm{mag}$ averaged over the $43 \mathrm{~m}$ telescope beam at position 1 . If $N_{p} / A_{V} \approx 1.4 \times 10^{21} \mathrm{~cm}^{-2} \mathrm{mag}^{-1}$ for the $\rho$ Oph region (Wilking \& Lada 1983), then $N_{p} \approx 7 \times 10^{22} \mathrm{~cm}^{-2}$ over the beam, nearly an order of magnitude higher than for the more standard dark cloud positions considered in Paper I. The beam-averaged volume density in the molecular gas toward position 1 is also likely to significantly higher. If the gas at this position is distributed along a line of sight dimension comparable to its dimension on the plane of the sky

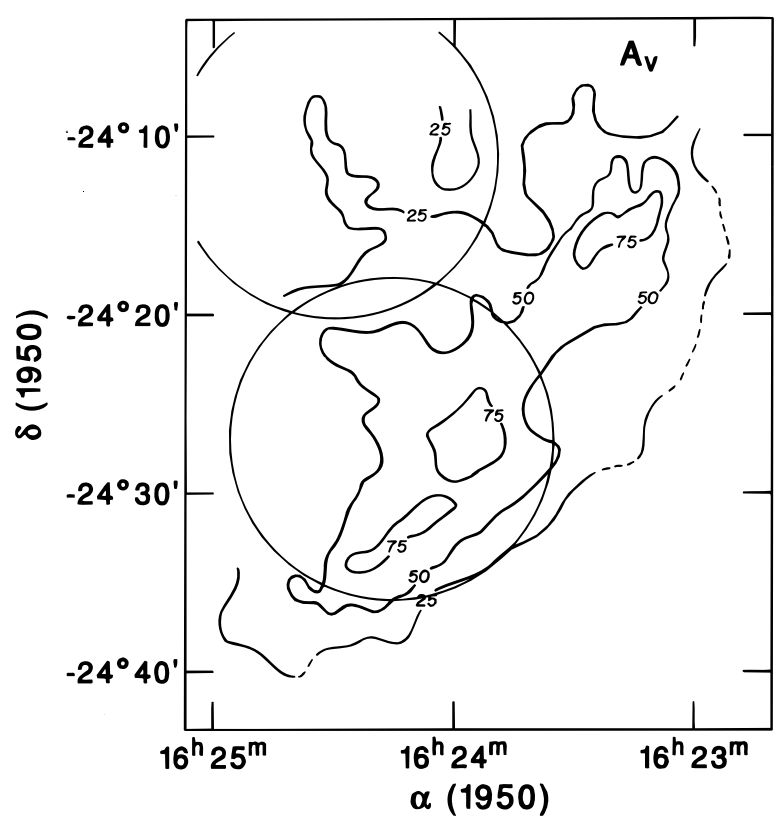

FIG. 3.-Map of $A_{v}$ toward the $\rho$ Oph core region taken from Wilking \& Lada (1983). This map is based upon the $\mathrm{C}^{18} \mathrm{O}$ data of these authors. The large circle near the center of the figure represents the FWHM of the NRAO $43 \mathrm{~m}$ telescope centered on $\rho$ Oph position 1 , the large circle at the upper left is for $\rho \mathrm{Oph}$ position 2 . 
( $\approx 20^{\prime}$ or $0.8 \mathrm{pc}$ ), then $n_{p} \approx 3 \times 10^{4} \mathrm{~cm}^{-3}$, again, about 10 times higher than for more standard dark clouds. Moreover, the $\rho$ Oph cloud contains even higher density gas on smaller scales. It is one of the few known regions where the $2 \mathrm{~cm} 2_{11}-2_{12}$ transition of $\mathrm{H}_{2} \mathrm{CO}$ is observed in emission, indicating densities of $10^{6} \mathrm{~cm}^{-3}$ or more (Martin-Pintado et al. 1983; Wadiak et al. 1985; Walmsley \& Wilson 1985). The $\mathrm{H}_{2} \mathrm{CO}$ emission comes from the $\rho \mathrm{Oph} \mathrm{B}$ region that is included in the beam area of our $\rho$ Oph position 1 .

In many ways, the $\rho$ Oph cloud is intermediate in nature between standard dark clouds that form low-mass stars and high-mass Orion-like molecular clouds that form massive stars and $\mathrm{H}$ II regions (Wootten 1991). Although OH emission line Zeeman observations of dark clouds usually yield field strengths or limits of only $\left|B_{\|}\right| \approx 10 \mu \mathrm{G}, \mathrm{OH}$ absorption line Zeeman studies of high-mass clouds frequently reveal fields of $\left|B_{\|}\right| \approx 100 \mu \mathrm{G}$ or more (see review by Troland 1990).

One additional characteristic of the $\rho$ Oph cloud is inferred from a comparison of ${ }^{12} \mathrm{CO},{ }^{13} \mathrm{CO}$, and $\mathrm{C}^{18} \mathrm{O}$ line profiles from this source (see Fig. 4). While the $\mathrm{C}^{18} \mathrm{O}$ line is very nearly symmetric about a velocity of about $3.7 \mathrm{~km} \mathrm{~s}^{-1}$, the ${ }^{12} \mathrm{CO}$ and ${ }^{13} \mathrm{CO}$ profiles are decidedly asymmetric, an asymmetry suggesting strong self absorption for velocities in the range $4-5 \mathrm{~km} \mathrm{~s}^{-1}$. This characteristic is consistent with an overall contraction of the $\rho$ Oph cloud at 1-2 km $\mathrm{s}^{-1}$ (Shu et al. 1987, and references therein).

\subsection{The Reduced Fractional Abundance of $\mathrm{OH}$ in the $\rho$ Oph Cloud}

Despite the comparatively high $N_{p}$ for the $\rho$ Oph cloud,

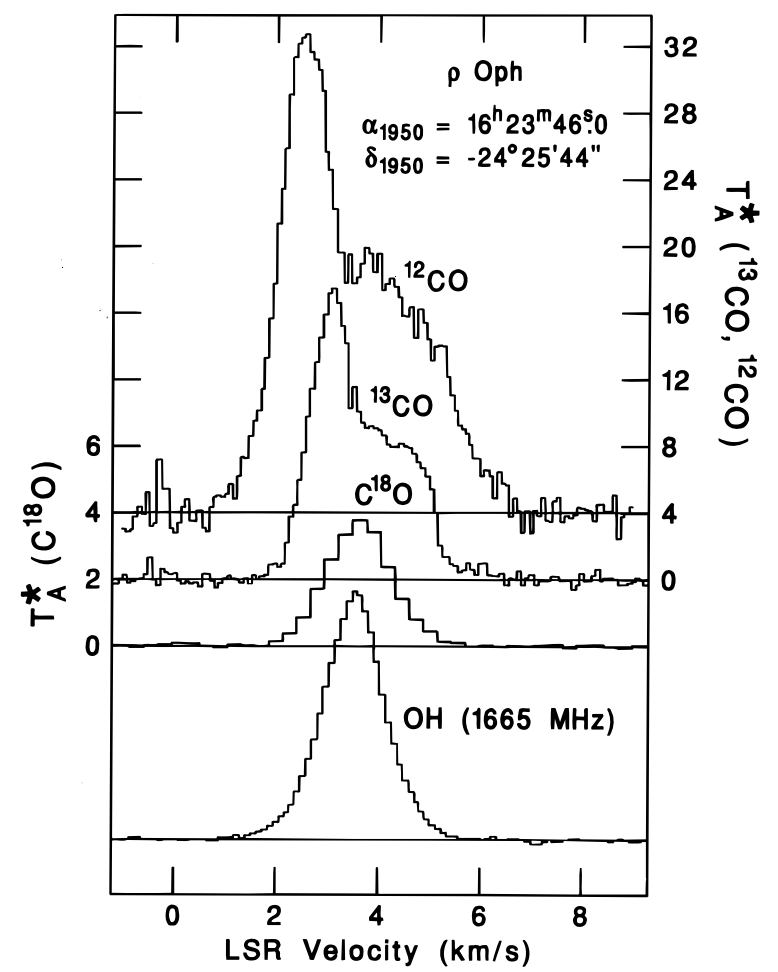

FIG. 4.- $\mathrm{CO}$ and $\mathrm{OH}$ profiles toward the $\rho \mathrm{Oph}$ core. The ${ }^{12} \mathrm{CO}$ and ${ }^{13} \mathrm{CO}$ profiles come from Wilking \& Lada (1983). They were obtained on the NRAO $11 \mathrm{~m}$ telescope with beam FWHM of 1'1, pointed at the position show on the figure of $\alpha_{1950} 16^{\mathrm{h}} 23^{\mathrm{m}} 46^{\mathrm{s}} .0, \delta_{1950}-24^{\circ} 25^{\prime} 44^{\prime \prime}$. The $\mathrm{C}^{18} \mathrm{O}$ profile was obtained recently in collaboration with D. A. Roberts on the NRAO $12 \mathrm{~m}$ telescope. This profile is the average of profiles obtained over a $14^{\prime} \times 14^{\prime}$ field centered on $\rho$ Ophiuchi position 1 . The $\mathrm{OH}$ profile is the $1667 \mathrm{MHz}$ profile of Fig. 1. the observed $\mathrm{OH}$ antenna temperatures and linewidths toward this source (hence, the estimated $N_{\mathrm{OH}}$ ) are comparable to or even smaller than those for the other (lower $N_{p}$ ) clouds listed in Table 2 of Paper I. This difference is almost certainly not a result of high $\mathrm{OH}$ optical depths in the $\rho$ Oph cloud. The $\mathrm{OH}$ antenna temperature ratio $T_{1665} / T_{1667} \approx 0.6$, implying $\tau_{1667} \approx 0.4$ if the two lines have the same $T_{\mathrm{ex}}$. Crutcher (1979) has shown that $T_{\mathrm{ex}}$ for the $\mathrm{OH}$ main lines in dark clouds may not be equal. However, if $\tau_{1667}>0.4$, then $T_{\text {ex }}<8 \mathrm{~K}$ in order to reproduce the observed $1667 \mathrm{MHz}$ line parameters. In view of the elevated temperatures in the $\rho$ Oph region, this possibility seems quite remote. Finally, the $\mathrm{OH}$ lines (Fig. 1) are exceptionally symmetric, and they display not the slightest trace of flattening at the top that might result from line saturation.

A much more likely possibility for the comparatively weak $\mathrm{OH}$ lines toward $\rho \mathrm{Oph}$ is a decline in $X_{\mathrm{OH}}$, the fractional abundance of $\mathrm{OH}$ inside the cloud. For the $\rho \mathrm{Oph}$ cloud, $A_{V}$ averaged over the $18^{\prime}$ telescope beam toward position 1 is about $50 \mathrm{mag}(\S 3.1)$. Also, $N_{\mathrm{OH}} \approx 6.4 \times 10^{14}$ $\mathrm{cm}^{-2}$ toward this position (Paper I, Table 2). Therefore, the ratio $N_{\mathrm{OH}} / A_{V} \approx 1.3 \times 10^{13} \mathrm{~cm}^{-2} \mathrm{mag}^{-1}$, or only $16 \%$ of the ratio reported by Crutcher (1979) for $0.4<A_{V}<7$.

Such a decline in $X_{\mathrm{OH}}$ in highly obscured clouds is consistent with theoretical expectations as reviewed by Heiles et al. (1993). $\mathrm{OH}$ formation is thought to occur as a result of ion-molecule reactions, a process that is limited by the ionization rate. For low $A_{V}$, starlight dominates the ionization processes, hence the formation (and destruction) of $\mathrm{OH}$. Under these conditions $X_{\mathrm{OH}}$ is expected to remain nearly independent of gas density. For higher $A_{V}$, ionization results from cosmic rays, and destruction, from reactions with neutral atoms. In this case, theoretical studies predict a declining $X_{\mathrm{OH}}$ with increasing gas density. The study by Leung, Herbst, \& Huebner (1984) suggests $X_{\mathrm{OH}} \propto n^{-0.8}$, while Langer \& Gradel (1989) find $X_{\mathrm{OH}} \propto n^{-0.7}$ after steady state is reached, with little dependence upon temperature. That is, $n_{\mathrm{OH}}$ is predicted to be nearly independent of total gas density at high $A_{V}$. Since much of the mass of the $\rho \mathrm{Oph}$ cloud has $A_{V} \gg 5 \mathrm{mag}$, the current $\mathrm{OH}$ line data provide observational support for the expected decline in $X_{\mathrm{OH}}$ in highly obscured regions.

\subsection{Densities Sampled by $\mathrm{OH}$ in the $\rho$ Oph Cloud Position 1}

A possible location for much of the $\mathrm{OH}$ in the $\rho \mathrm{Oph}$ cloud is in low-density envelope gas external to the dense ridge defined by $\mathrm{C}^{18} \mathrm{O}$ emission (Fig. 3). Recall that in this ridge $n_{p} \approx 3 \times 10^{4} \mathrm{~cm}^{-3}$ at position 1 averaged over the $18^{\prime}$ beam of the telescope ( $\$ 3.1)$. While this possibility cannot be completely excluded, it seems quite unlikely for two reasons. First, the higher spatial resolution $\left(8^{\prime}\right.$ beam width) $\mathrm{OH}$ map of Myers et al. (1978) suggests a ridgelike morphology, much like the distribution of $\mathrm{C}^{18} \mathrm{O}$ emission and unlike that of ${ }^{12} \mathrm{CO}$ or ${ }^{13} \mathrm{CO}$ (see the maps of Loren \& Wootten 1986). Second, the $\mathrm{OH}$ line shape and center velocity for position 1 match extremely well those of the $\mathrm{C}^{18} \mathrm{O}$ line for a similar region of sky. This correspondence is shown in Figure 4 where the $\mathrm{C}^{18} \mathrm{O}$ profile is an average of profiles obtained by the NRAO $12 \mathrm{~m}$ telescope over a $14^{\prime} \times 14^{\prime}$ field centered on position 1 . Furthermore, the $\mathrm{OH}$ line center velocity and width are very similar to those of other high density tracers like the $J=3-2 \mathrm{CS}$ lines toward $\rho$ Oph cores B1 and B2 (both within the $\mathrm{OH}$ beam at position 1), the $J_{K}=6_{5}-5_{4}$ lines of SO toward B1 and B2, and 
the $\mathrm{DCO}^{+}$line toward $\mathrm{B} 2$ (see profiles in Loren \& Wootten 1986). However, the ${ }^{13} \mathrm{CO}$ and especially the ${ }^{12} \mathrm{CO}$ lines toward the dense ridge of gas defined by $\mathrm{C}^{18} \mathrm{O}$ emission are significantly wider than the $\mathrm{OH}$ lines (Fig. 4). The most straightforward interpretation of all these data is that the $\mathrm{OH}$ line flux arises principally within the volume of space occupied by the ridge of high density gas. However, this interpretation does not necessarily mean that the $\mathrm{OH}$ emission samples the dense gas. Another possibility is that the ridge is highly clumped, with $\mathrm{OH}$ emission arising mostly in a lower density interclump medium.

\subsection{The Magnetic Field in $\mathrm{H}$ I Gas Near the $\rho$ Oph Cloud}

Goodman \& Heiles (1994) have recently reported magnetic field measurements at a number of positions in the $\rho$ Oph region based upon the $\mathrm{H}$ I Zeeman effect. Their position 15 corresponds to our $\mathrm{OH}$ Zeeman position 1, although the beam of the Hat Creek 85' telescope used for the $\mathrm{H}$ I observations is about two times larger in diameter. Goodman \& Heiles performed a Gaussian analysis of the $\mathrm{H}$ I emission line profile at this and other positions, identifying a component in self-absorption. At position 15 they report a Zeeman effect in this component equivalent to $B_{\|}=+13 \pm 1.7 \mu \mathrm{G}$, a figure of comparable absolute value but opposite sign to that derived from our $\mathrm{OH}$ data for position 1. At seven other positions within about a degree of position 1 , Goodman \& Heiles report detections of the field in $\mathrm{H}$ I self-absorption in the range $+5<B_{\|}<+12 \mu \mathrm{G}$, while at about the same number of positions in this area they either find no detectable field in $\mathrm{H}$ I self-absorption or no self-absorption component at all. From an analysis of the $\mathrm{H}$ I Zeeman data together with optical linear polarization data, they estimate that the uniform field component in the $\mathrm{H}$ I self-absorbing region toward the $\rho$ Oph cloud is about $+10 \mu \mathrm{G}$ with an inclination to the line of sight of about $30^{\circ}$.

It is quite probable that these $\mathrm{H}$ i measurements refer to inerstellar gas closely associated with the molecular gas toward the $\rho$ Oph cloud. Although the spatial distribution of $\mathrm{H}$ I self-absorption does not match that of the molecular gas as traced out by any CO isotopic species (see, for example, maps in Loren \& Wootten 1986) or by OH (Myers et al. 1978), the line widths and center velocities appear to match rather well. For example, at Goodman \& Heiles position 15 , the $\mathrm{H}$ I self absorption component they identify has a center velocity and velocity width of 4.9 and $1.5 \mathrm{~km}$ $\mathrm{s}^{-1}$, respectively. This width matches very well those of the $\mathrm{OH}$ lines $\left(1.4 \mathrm{~km} \mathrm{~s}^{-1}\right)$, while the $\mathrm{H} \mathrm{I}$ center velocity is about $1 \mathrm{~km} \mathrm{~s}^{-1}$ more positive. The amount and sign of this difference in center velocities is consistent with the location of the cold $\mathrm{H}$ i directly in front of the molecular cloud, adjacent to the ${ }^{12} \mathrm{CO}$ and ${ }^{13} \mathrm{CO}$ self absorption region (\$ 3.1 and Fig. 4). At the $\mathrm{H}$ I center velocity of $4.9 \mathrm{~km} \mathrm{~s}^{-1}$, the $\mathrm{OH}$ line temperature is very low (Figs. 1 or 4). Most probably, the $\mathrm{H} \mathrm{I}$ field of order $10 \mu \mathrm{G}$ measured by Goodman \& Heiles characterizes an envelope region just in front of the denser molecular gas sampled by the OH Zeeman effect. However, it should be noted that there are significant uncertainties in the derived parameters for $\mathrm{H}$ I self absorption toward $\rho$ Oph. For example, Minn's (1981) study with the MaxPlanck $100 \mathrm{~m}$ telescope yields an $\mathrm{H}$ i self absorption center velocity at $\mathrm{OH}$ Zeeman position 1 of 3.5 to $4 \mathrm{~km} \mathrm{~s}^{-1}$ (his Fig. 4), while a similar study by Myers et al. (1978) with the $43 \mathrm{~m}$ telescope yields an $\mathrm{H}$ I center velocity and velocity width of $3.5 \mathrm{~km} \mathrm{~s}^{-1}$ and $2.8 \mathrm{~km} \mathrm{~s}^{-1}$, respectively, for the same position (their Fig. 8). These differences may reflect differences in the fitting processes used by various authors or else differences in telescope beamwidth.

\subsection{The Magnetic Field in $\mathrm{OH}$ Gas in the $\rho$ Oph Cloud}

It is notable that $\left|B_{\|}\right|$from the $\mathrm{OH}$ Zeeman data is comparable to (possibly even less than) that from the $\mathrm{H} \mathrm{I}$ Zeeman data even though $\mathrm{H}$ I surely samples a much lower density region. Goodman \& Heiles estimate a proton density $n_{p} \approx 40 \mathrm{~cm}^{-3}$ in the $\mathrm{H}$ I self absorbing gas, while on the average $n_{p} \approx 3 \times 10^{4} \mathrm{~cm}^{-3}$ in the molecular gas encompassed by the $18^{\prime}$ telescope beam toward position $1(\S 3.1)$. One might well expect a measurable contrast in field strength given such a density contrast of order $10^{3}$.

Higher $\mathrm{OH}$ field strengths are expected in the $\rho \mathrm{Oph}$ cloud on two other grounds. For one, if magnetic and gravitational energies are equal in the cloud, then $|\boldsymbol{B}|=$ $|\boldsymbol{B}|_{\text {virial }} \approx 2.4 \times 10^{-21} \mathrm{k} N_{p} \mu \mathrm{G}$. (See Paper I and references therein.) In this relation, $k \approx 2$ for clouds that have evolved from initial states of uniform density and magnetic field, and $k$ is close to unity for any realistic cases. Therefore, the beam averaged $N_{p}$ cited above implies $|\boldsymbol{B}| \approx 300 \mu \mathrm{G}$ toward position 1 . This is the mean field strength required to support the cloud against gravitation in the absence of other sources of support. Alternatively, if the kinetic energy density in the cloud associated with nonthermal broadening $\Delta v_{\mathrm{NT}}$ of the $\mathrm{OH}$ line (or of various millimeter wavelength lines) equals the magnetic energy density, then $|\boldsymbol{B}|=$ $|\boldsymbol{B}|_{\mathrm{A}} \approx 0.4 \Delta v_{\mathrm{NT}} n_{p}^{1 / 2} \approx 100 \mu \mathrm{G} .{ }^{9}$ This assumption amounts to having $\Delta v_{\mathrm{NT}} \approx v_{\mathrm{A}}$, the Alfvén velocity in the cloud; that is, assuming that the nonthermal component of the line width is a result of Alfvén waves. Observations also suggest that this criterion is at least approximately met in most molecular clouds (Myers \& Goodman 1988; Paper I). Note that the estimates here for $|\boldsymbol{B}|_{\text {virial }}$ and $|\boldsymbol{B}|_{\mathrm{A}}$ are higher than those in Paper I, Table 2 because we have chosen higher values of $N_{p}$ and $n_{p}$ derived from $\mathrm{C}^{18} \mathrm{O}$ rather than $\mathrm{OH}$ lines. These higher values reflect the actual (beam-averaged) conditions in $\rho$ Oph position 1 , not the conditions inferred from the emission of underabundant $\mathrm{OH}$.

The weakness of $B_{\|}$implied by the OH Zeeman observations can be viewed in terms of two limiting cases. In one case $|\boldsymbol{B}|$ is assumed to be quite high in the sampled regions of the cloud $(\geq 100 \mu \mathrm{G})$, comparable to field strengths estimated above from virial and also from line width considerations. In this "high field" case, the low measured $B_{\|}$must result from very significant geometric effects. For example, a relatively unifom $\boldsymbol{B}$ with $|\boldsymbol{B}| \geq 100 \mu \mathrm{G}$ might permeate the cloud but be oriented nearly in the plane of the sky. However, to achieve $B_{\|} \leq 0.1|\boldsymbol{B}|$ we require a rather unlikely field alignment to within about $5^{\circ}$ of the plane of the sky, an alignment that presumably does not apply to the field in the adjacent $\mathrm{H}$ I gas. Alternately, significant reversals of the field might exist within the beam. The possibility of such reversals is strongly suggested by the disorder in optical (Vrba, Strom, \& Strom 1976) and near-IR (Sato et al. 1988) linear polarization vectors toward the $\rho$ Oph cloud. Field reversals are also suggested by the difference in sign of $B_{\|}$as measured in $\mathrm{OH}$ and $\mathrm{H}$ I regions.

In the other limiting case $|\boldsymbol{B}|$ is assumed to be quite low

\footnotetext{
9 This equation is equivalent to equation (6) of Myers \& Goodman (1988) with a correction factor included for the mass contribution of $\mathrm{He}$, and it is the equation used in Paper I to compute $|\boldsymbol{B}|_{\mathrm{A}}$
} 
in the sampled regions of the cloud $(\ll 100 \mu \mathrm{G})$, wherever those sampled regions may be. In this "low field" case, significant geometric effects are not needed to explain the small measured value of $B_{\|}$. However, if such low field strengths characterize much of the gas of the $\mathrm{C}^{18} \mathrm{O}$ ridge, then the field there is incapable of supporting the ridge against self-gravitation. In this situation, the ridge is likely to be in a state of magnetically supercritical collapse, perhaps at a speed of $1-2 \mathrm{~km} \mathrm{~s}^{-1}$ as suggested by Shu et al. (1987) on the basis of the self-absorption in ${ }^{12} \mathrm{CO}$ and ${ }^{13} \mathrm{CO}$ lines. (Such a collapse would likely cause significant field tangling, hence disorder in optical and near-IR linear polarization vectors, as observed.) So low a field in so dense a region would also imply that the motions responsible for the nonthermal contribution to the line widths are superAlfvénic. Since super-Alfvénic motions in a cloud are expected to be highly dissipative, their presence in the $\rho$ Oph ridge might reflect active sources of mechanical energy. Alternately, the motions responsible for the observed line widths may reflect the collapse motions themselves.

The other obvious possibility in the low field case is that the $\mathrm{OH}$ lines sample densities in the $\mathrm{C}^{18} \mathrm{O}$ ridge much lower than the average value of about $3 \times 10^{4} \mathrm{~cm}^{-3}(\S 3.2)$. If the $\mathrm{OH}$ line flux comes mostly from lower density gas in the ridge (density comparable to that sampled in the standard dark clouds of Paper I), then the measured value of $B_{\|}$ might well be comparable to those of the other clouds. Even so, the density contrast between gas sampled by $\mathrm{OH}$ and $\mathrm{H}$ I toward $\rho \mathrm{Oph}$ is almost certainly at least 2 orders of magnitude.

A large density contrast need not necessarily be accompanied by a significant contrast in field strength if some recent theoretical formulations are correct. If the $\rho \mathrm{Oph}$ cloud formed by ambipolar diffusion within a magnetically supported envelope, as modeled for the dark cloud Barnard 1 by Crutcher et al. (1994), then the field strength throughout much of the $\rho$ Oph ridge should not have increased appreciably in the process. In the Barnard 1 evolutionary model of Crutcher et al., the envelope field strength increases by less than a factor of 2 over the assumed background value while the envelope density becomes 2 orders of magnitude greater than its background value. Note, however, that if $\mathrm{OH}$ field strengths in the $\rho \mathrm{Oph}$ ridge are small because the lines originate principally in lower density gas, then the field must still be hundreds of microGauss in the undersampled high density gas if the field is to provide appreciable support to the ridge against self-gravitation.

\section{THE TMC-1C CLOUD CORE}

\subsection{The Nature of the TMC-1C Core}

The TMC-1C core is one of numerous dense cores in the Taurus complex that have been studied in $\mathrm{CO}$ by Myers, Linke, \& Benson (1983) and in $\mathrm{NH}_{3}$ by Myers \& Benson (1983), Benson \& Myers (1989), and by Goodman et al. (1993). These cores are at a distance of about $140 \mathrm{pc}$ (Elias 1978). The TMC-1C $\mathrm{NH}_{3}$ core is elongated, with dimensions $6^{\prime} \times 2^{\prime}(0.25 \times 0.09 \mathrm{pc}$ at a distance of $140 \mathrm{pc})$, and it has a small velocity gradient along its long dimension of $0.30 \pm 0.07 \mathrm{~km} \mathrm{~s}^{-1} \mathrm{pc}^{-1}$ (Goodman et al. 1993). The $\mathrm{NH}_{3}$ data of Myers \& Benson (1983) suggest $n_{p} \approx 2 \times 10^{4} \mathrm{~cm}^{-3}$, while $\mathrm{C}^{18} \mathrm{O}$ observations of Myers \& Benson (1983) yield an estimate $N_{p} \approx 2 \times 10^{22} \mathrm{~cm}^{-2}$. Thus, $A_{V} \approx 10$ through this core, very comparable to that of Barnard 1 (Bachiller \&
Cernicharo 1984) and other cloud positions reported upon in Paper I except for the $\rho$ Oph cloud. With its $3^{\prime}$ beam, the Arecibo telescope is well matched to the dimensions of the dense core, unlike the case of the $18^{\prime}$ beam of the $43 \mathrm{~m}$ telescope. Moreover, the close proximity of the Taurus complex $(\approx 140 \mathrm{pc})$ means that the linear scale sampled by the Arecibo beam is only about $0.1 \mathrm{pc}$, the smallest linear scale studied so far in single dish Zeeman effect studies of dark clouds.

\section{2. $\mathrm{OH}$ in the TMC-1C Core}

The $\mathrm{OH}$ lines from the TMC-1C core are very narrow $\left(\Delta v \approx 0.44 \mathrm{~km} \mathrm{~s}^{-1}\right)$, narrower than any of the $\mathrm{OH}$ lines cited in Paper I. This width is also less than the $1.2 \mathrm{~km} \mathrm{~s}^{-1}$ TMC-1C ${ }^{13}$ CO line width reported by Myers et al. (1983) with a $1^{\prime} .9$ beam, but is virtually identical to the $\mathrm{C}^{18} \mathrm{O}$ line width $\left(0.47 \mathrm{~km} \mathrm{~s}^{-1}\right)$ given by these same authors. (TMC-1C shares this similarity between $\mathrm{OH}$ and $\mathrm{C}^{18} \mathrm{O}$ line widths with $\rho$ Oph position 1.) The $\mathrm{NH}_{3}$ line from TMC-1C is narrower yet, $\Delta v \approx 0.24 \mathrm{~km} \mathrm{~s}^{-1}$ observed with a 1.4 beam (Benson \& Myers 1989).

If $N_{\mathrm{OH}}$ is estimated in the manner of Paper I, and $T_{\mathrm{ex}}=10$ $\mathrm{K}$ (Benson \& Myers 1989), then $\tau_{1667}=0.45$ and $N_{\mathrm{OH}} \approx 5$ $\times 10^{14} \mathrm{~cm}^{-2}$. With $A_{V} \approx 10 \mathrm{mag}(\S 4.1)$, this value of $N_{\mathrm{OH}}$ implies $N_{\mathrm{OH}} / A_{v}=5 \times 10^{13} \mathrm{~cm}^{-2} \mathrm{mag}^{-1}$, about $60 \%$ of the ratio reported by Crutcher (1979). Evidently, $\mathrm{OH}$ is not significantly depleted in TMC-1C as it appears to be in $\rho$ Oph position 1. This conclusion is consistent with the significantly lower $A_{v}$ toward TMC-1C and with the theoretically expected effect of lower $A_{v}$ upon the ionization rate, hence upon the $\mathrm{OH}$ abundance (\$3.2). Since $\mathrm{OH}$ is not significantly depleted in TMC-1C, and since the Arecibo beam is comparable in dimension to the TMC-1C core, it is likely that a significant fraction of the $\mathrm{OH}$ line emission originates in the relatively high density $\left(n_{p} \approx 10^{4} \mathrm{~cm}^{-3}\right)$ core.

\subsection{The Magnetic Field in TMC-1C}

Güsten \& Fiebig (1990) originally reported the detection of the Zeeman effect in the $11.12 \mathrm{GHz}$ CCS $\left(1_{0}-0_{1}\right)$ ground state transition from TMC-1C. They derived $B_{\|} \approx-100$ $\mu \mathrm{G}$, and this result was the principal motivation for the $\mathrm{OH}$ Zeeman observation reported here. Subsequently, instrumental effects were identified as the source of the apparent Zeeman effect (Güsten 1990, private communication). However, a field strength of this magnitude is not unexpected in the TMC-1C core. Given the column density estimated for this region, $|\boldsymbol{B}|_{\text {virial }} \approx 100 \mu \mathrm{G}$ (see $\S 3.5$ ). This value is more than 10 times larger than the $3 \sigma$ upper limit on $B_{\|}$of $8.4 \mu \mathrm{G}$ implied by the $\mathrm{OH}$ Zeeman observations (§ 2).

As in the case of the $\rho$ Oph cloud, we can imagine two extremes for the magnetic field in the TMC-1C core. In the low field case, $|\boldsymbol{B}| \leq 10 \mu \mathrm{G} \ll|\boldsymbol{B}|_{\text {virial }}$. If so, the core is magnetically supercritical. It cannot be supported by the magnetic field, and it could conceivably be in a state of supercritical collapse. However, the narrow $\mathrm{OH}$ and especially $\mathrm{NH}_{3}$ line widths limit the speed of such a collapse if it is presently occurring, and there is no other evidence for collapse as there is for the $\rho$ Oph cloud. For these reasons, this possibility seems quite unlikely.

In the high field case, $|\boldsymbol{B}|=|\boldsymbol{B}|_{\text {virial }} \approx 100 \mu \mathrm{G}$, and the measured $\boldsymbol{B}_{\|} \ll|\boldsymbol{B}|$ either because of geometrical factors including field reversal or else because of high $\mathrm{OH}$ optical 
depths that result in poor sampling of the dense core gas. Moreover, $|\boldsymbol{B}| \gg|\boldsymbol{B}|_{\mathrm{A}} \approx 25 \mu \mathrm{G}$, given the $\mathrm{OH}$ line width and given the density estimated from $\mathrm{NH}_{3}$ data $(\S 4.1)$. That is, the line widths are significantly less than the Alfvén speed, or equivalently, the energy density associated with the non-thermal line widths is much less than that of the magnetic field. Such a cloud is magnetically supported more by static field pressure than by motions associated with Alfvén waves. Since static magnetic support occurs only perpendicular to the field direction, a cloud so supported should be flattened into a disk perpendicular to the field. In fact, the TMC-1C $\mathrm{NH}_{3}$ core is elongated, its 3:1 aspect ratio is one of the highest observed by Benson \& Myers (1989) in a study of over 40 cores. Yet this elongation is not likely to result from rotation alone, since Goodman et al. (1993) found the core to have one of the smallest velocity gradients detected in many of the same cores considered by Benson \& Myers. If the elongation of the TMC-1C core is the result of flattening along magnetic field lines, then the relatively high aspect ratio implies that the symmetry axis of the cloud (hence the magnetic field) lies at a rather large angle to the line of sight, hence $B_{\|} \ll|B|$.

\section{CONCLUSIONS}

The dark cloud positions discussed here provide additional insights into magnetic fields, especially those in molecular gas sampled by the $\mathrm{OH}$ Zeeman effect. The results for the $\rho \mathrm{Oph}$ cloud and for the dark cloud core TMC-1C underscore how $\mathrm{OH}$ Zeeman observations of dark clouds reveal relatively weak magnetic fields (of order $10 \mu \mathrm{G}$ or less, Paper I). These fields are weak in the sense that they are comparable to field strengths in much lower density regions revealed by the $\mathrm{H}_{\text {I }}$ Zeeman effect. Such weak fields apparently exist even for the $\rho$ Oph cloud position 1 with its high column and volume densities and even for the TMC-1C core for which we resolve small spatial scales $(0.1 \mathrm{pc})$. Moreover, with the possible exception of the $\rho$ Oph cloud, weak fields derived from $\mathrm{OH}$ Zeeman observations are unlikely to reflect poor sampling of the dark cloud mass by the $\mathrm{OH}$ lines owing either to declining fractional $\mathrm{OH}$ abundance or to $\mathrm{OH}$ optical depth effects.

Despite the weakness of fields derived from the $\mathrm{OH}$ Zeeman effect, the results are still consistent in most cases with the idea that dark clouds are in approximate equilibrium between magnetic and gravitational energies (Paper I). One context in which this equilibrium can be understood involves static magnetic support of dark cloud envelopes and the formation of dark cloud cores via ambipolar diffusion, as modeled for Barnard 1 by Crutcher et al. (1994). A similar evolutionary process may have characterized dark cloud core TMC-1C. In both cases, the mean magnetic field may lie at a large angle to the line of sight, hence $B_{\|} \ll|\boldsymbol{B}|$. But such unfortunate geometry cannot characterize all dark cloud cores. The Barnard 1 model calls for an easily detectable $85 \mu \mathrm{G}$ field in the core. If Barnard 1 is typical and the model is correct, then future $\mathrm{OH}$ Zeeman observations of dark cloud cores with an Arecibo-sized beam should yield a number of detections.

The $\rho$ Oph cloud may well fall into a separate category. If field strengths in this cloud are truly comparable to $B_{\|}$ derived from the $\mathrm{OH}$ Zeeman effect, then the region may be magnetically supercritical, and it may at present be undergoing supercritical collapse, an apparently unusual and highly interesting state. Alternately, much of the gas in the $\rho$ Oph cloud may exist in a high density regime for which the fractional abundance of $\mathrm{OH}$ is greatly reduced. In this case, most of the $\mathrm{OH}$ line flux originates in a lower density envelope region, and the measured value of $B_{\|}$applies to this region alone. If so, the similarity in field values implied by $\mathrm{OH}$ and $\mathrm{H}$ I Zeeman effect observations may reflect the very weak connection between field strength and gas density expected in magnetically supported envelopes of self-gravitating clouds as outlined in the model for Barnard 1 of Crutcher et al. (1994).

We wish to thank D. A. Roberts for his assistance in obtaining the $\mathrm{C}^{18} \mathrm{O}$ spectrum of Figure 4. This work was supported in part by NSF grants AST 91-16917, INT 9116356, AST 94-19220, and AST 94-19227.
Bachiller, R., \& Cernicharo, J. 1984, A\&A, 140, 414

Benson, P. J., \& Myers, P. C. 1989, ApJS, 71, 89

Crutcher, R. M. 1979, ApJ, 234, 881

Crutcher, R. M., Mouschovias, T. Ch., Troland, T. H., \& Ciolek, G. E. 1994, ApJ, 427, 839

Crutcher, R. M., Troland, T. H., Goodman, A. A., Heiles, C., Kazes, I., \& Myers, P. C. 1993, ApJ, 407, 175 (Paper I)

de Geus, E. J., \& Burton, W. B. 1991, A\&A, 246, 559

de Geus, E. J., de Zeeuw, P. T., \& Lub, J. 1989, A\&A, 216, 44

Elias, J. H. 1978, ApJ, 224, 857

Goodman, A. A., Benson, P. J., Fuller, G. A., \& Myers, P. C. 1993, ApJ 406, 528

Goodman, A. A., Crutcher, R. M., Heiles, C., Myers, P. C., \& Troland, T. H. 1989, ApJ, 338, L61

Goodman, A. A., \& Heiles, C. 1994, ApJ, 424, 208

Güsten, R. F., \& Fiebig, D. 1990, in IAU Symp. 140, Galactic and Intergalactic Magnetic Fields, ed. R. Beck, P. P. Kronberg, \& R. Wielebinsk (Dordrecht: Kluwer), 305

Heiles, C., Goodman, A. A., McKee, C. F., \& Zweibel, E. G. 1993, in Protostars and Planets III, ed. E. H. Levy \& J. I. Lunine (Tucson: Univ. Arizona Press), 279

Lada, C. J., \& Wilking, B. A.1984, ApJ, 287, 610

Langer, W. D. \& Graedel, T. E. 1989, ApJS, 69, 241

Leung, C. M., Herbst, E., \& Huebner, W. F. 1984, ApJS, 56, 231

Loren, R. B., Sandquist, A., \& Wootten, A. 1983, ApJ, 270, 620

Loren, R. B., \& Wootten, A. 1986, ApJ, 306, 142

Martín-Pintado, J., Wilson, T. L., Gardner, F. F., \& Henkel, C. 1983, A\&A 117,145

McKee, C. F., Zweibel, E. G., Goodman, A. A., \& Heiles, C. 1993, in Protostars and Planets III, ed. E. H. Levy \& J. I. Lunine (Tucson: Univ. Arizona Press) 327

\section{REFERENCES}

Minn, Y. K. 1981, A\&A, 103, 269

Mouschovias, T. Ch. 1987, in Physical Processes in Interstellar Clouds, ed. G. Morfil \& M. Scholer (Dordrecht: Reidel), 453

1991a, in The Physics of Star Formation, ed. C. J. Lada \& N. D. Kylafis (Dordrecht: Kluwer), 61

. 1991b, in The Physics of Star Formation, ed. C. J. Lada \& N. D. Kylafis (Dordrecht: Kluwer), 449

Myers, P. C., \& Benson, P. J. 1983, ApJ, 266, 309

Myers, P. C., \& Goodman, A. A. 1988, ApJ, 329, 392

Myers, P. C., Ho, P. T. P., Schneps, M. H., Chin, G., Pankonin, V., \& Winnberg, A. 1978, A\&A, 220, 864

Myers, P. C., Linke, R. A., \& Benson, P. J. 1983, ApJ, 264, 517

Roberts, D. A., Crutcher, R. M., \& Troland, T. H. 1995, ApJ, 442, 208

Sato, S., Tamura, M., Nagata, T., Kaifu, N., Hough, J., McLean, I. S., Garden, R. P., \& Gatley, I. 1988, MNRAS, 230, 321

Shu, F. H., Adams, F. C., \& Lizano, S. 1987, ARA\&A, 25, 23

Troland, T. H. 1990, in IAU Symp. 140, Galactic and Intergalactic Magnetic Fields, ed. R. Beck, P. P. Kronberg, \& R. Wielebinski (Dordrecht: Kluwer), 293

Vrba, F. J., Strom, S. E., \& Strom, K. M. 1976, AJ, 81, 958

Wadiak, E. J., Wilson, T. L., Rood, R. T., \& Johnston, K. J. 1985, ApJ, 295 L43

Walmsley, C. M., \& Wilson, T. L. 1985, in Proceedings of a Specialized Colloquium of the Eighth IAU European Regional Astronomy Meeting, Nearby Molecular Clouds, (Berlin: Springer), 41

Wilking, B. A., \& Lada, C. J. 1983, ApJ, 274, 698

Wootten, A. 1991, in IAU Symp. 147, Fragmentation of Molecular Clouds and Star Formation, ed. E. Falgarone, F. Boulanger, \& G. Duvert (Dordrecht: Kluwer), 229

Zeng, Q., Batrla, W., \& Wilson, T. L. 1984, A\&A, 141, 127 